\title{
Chest drain care bundle: Improving documentation and safety
}

\author{
Joe Hutton, Selina Graham \\ Gloucestershire NHS Trust, UK
}

\begin{abstract}
Chest drain insertion is a common advanced procedure with a significant associated risk of pain, distress, and complications. Nationally, audit and recommendations from leading bodies have highlighted a number of safety concerns around chest drain insertion.
\end{abstract}

Audit work has demonstrated poor levels of documentation; particularly around use of premedication, use of ultrasound guidance and consent. This has obvious potential consequences for patient safety and thus is an important target for improvement work.

This project quantifies current standards of documentation and aims to improve this through a combination of accessible and easy to read guidelines, education, and the introduction of a chest drain insertion bundle. National best practice standards were identified through review of national guidance.

Drain insertion was prospectively analysed over a three month period to establish baseline standards of documentation. This initial work was presented and a bundle and clinical guidelines produced. Chest drain insertion was then reaudited and assessed for improvement.

Results demonstrated an improvement in many areas of documentation, pushing local results above the national average. However, only $40 \%$ of cases used the new bundle due to a mixture of staff rotation and an unexpectedly high proportion of drains inserted in non targeted areas including the emergency department, theatre, and intensive care. Despite this, the introduction of accessible guidance and bundle has significantly improved chest drain insertion documentation to the benefit of all.

\section{Problem}

The insertion of chest drains is a relatively common advanced procedure in hospital; especially in hospitals with busy acute medical takes and respiratory units. However, the insertion of chest drains can cause significant pain and distress in patients [1] and can cause significant complications [2]. Some of these complications can be potentially fatal [2]. As such, proper documentation of drain insertion and consent is paramount.

Prior to this work, insertion and documentation of chest drains in Gloucester NHS Trust, United Kingdom (UK) had not been examined. Similarly, no hospital approved guidance or insertion bundle existed for this important procedure. This may have contributed to inadequate documentation and potential patient safety incidents. In addition, chest drains are often inserted in areas of high staff turnover such as the emergency department or acute care unit. This can create difficulty for staff taking over patient care to find out details of the procedure and follow up outstanding requested investigations. There were also concerns that not all practitioners who were inserting drains were fully aware of the most current guidance from the British Thoracic Society on best practice in this area; particularly around the use of ultrasonography and premedication. These factors highlighted the documentation of chest drain insertion as an area for improvement important for patient safety.

\section{Background}

Chest drains are commonly inserted for the management and further investigation of chest pathology. Indications include pleural effusion, pneumothorax, haemothorax, and post-thoracic surgery. For medical chest drains, the Seldinger technique is typically used with the requisite equipment provided in prefabricated packs.

Concerns regarding correct chest drain insertion documentation and patient consent have been raised by both the National Patient Safety Agency and the British Thoracic Society [3]. Indeed, the national audit of chest drains [4] flagged up a number of safety concerns around the insertion of chest drains, particularly around access to ultrasonography and training for this vital skill. Other areas of concern included lack of documentation of informed consent in $42 \%$ of cases. It also highlighted that the majority of drains were inserted at the patient bedside despite active discouragement from current guidance [3]. Other problem areas included indication for chest drain, with many drains being inserted for undiagnosed pleural effusions, potentially delaying diagnosis in patients [4]. The BTS list in their guidance on pleural procedures the things that should be documented prior to chest drain insertion [5] and national audit is increasingly a priority.

Following the British Thoracic Society's guidance on chest drain insertion would allow minimisation of the risk of complications and has been demonstrated to reduce patient anxiety and pain [2].

Care bundles have been widely used in many other areas of medicine and surgery to improve documentation and practice [6]. These have been demonstrated to improve practice and patient 
BMJ Quality Improvement Reports

outcomes in many areas of medicine; including intensive care and respiratory medicine [6-8]. Care bundles can improve patient survival rates, reduce length of stay, and reduce risk of some infections. As such, use of a similar bundle for chest drain insertion offers an appealing method of improving practice.

Care bundles also have several other functions in aiding best practice. They provide a prompt to the clinician by reminding them of specific steps advised in performing best practice. They also aid consistency in service delivery and enable ease of documentation. By using a uniform method of presentation and format designed to ensure all important points are recorded, they make it much easier for other team members to review the procedure, follow-up outstanding results, and ensure the best care possible is delivered.

\section{Baseline measurement}

A prospective audit of patients with newly inserted chest drains on the acute care unit, respiratory unit, and oncology was undertaken over a period of three months. Posters advertising the project and data collection forms were posted in these locations and staff working in these areas approached to identify potential cases.

Data was collected on various audit measures following review of the best practice advised by the British Thoracic Society [5]. A full list of these is included in the following section and data collection tool attached (Appendix 1). The gathered results were anonymised. Records of patient identifiable details were kept on a separate data collection tool and kept in a secure location. The standards for each of the audit measures would ideally be $100 \%$. This data was analysed and areas for potential improvement highlighted.

The results showed that the date was recorded in $91.7 \%$ of cases, but that the time was only documented for $58.3 \%$. The indication was reported in $100 \%$. Only $16.7 \%$ of cases received premedication. In cases where patients did not receive premedication, in no cases was the rationale for not giving this medication given. Consent was only recorded in $66.7 \%$ of total patients. $68.8 \%$ of those who had consent recorded had formal written consent. Worryingly, only $50 \%$ of drains were undertaken with ultrasound guidance. The drain site was recorded in $41.7 \%$. Local anaesthetic use was recorded in $66.7 \% .54 .2 \%$ recorded the volume of local anaesthetic used.

Vitals details of drain insertion such the length inserted to was recorded in $17 \%$, the size of the drain in $58.3 \%$ and how the drain was secured in $41.7 \%$. Post procedure advice was recorded in $66.7 \%$ of cases. Drain observation sheets were used in $79.2 \%$. $87.5 \%$ of patients had the interpretation of post-drain insertion chest radiograph documented in their notes. There was a written record of pleural samples sent for testing in $54.2 \%$ of cases.

This initial work highlighted several key areas for improvement. Some of these were around simple measures that should be recorded in any clinical interaction. However, particular areas for improvement were demonstrated in the use of ultrasound guidance, pre-medication, patient consent, and specific details of the insertion and investigations requested.
See supplementary file: ds6007.pdf - "appendices"

\section{Design}

The initial audit was presented to the Respiratory department including the Trust lead for Pleural Procedures. It was concluded that documentation levels were largely poor and none of the recorded examples included all of the key points recommended by the BTS. This therefore raised a crucial need for improvement. Care bundles are widely used in medicine and thus the trial of a chest drain care bundle was agreed. Members of the respiratory team and the BTS guidance was used to inform which areas were important to include in documentation.

\section{Strategy}

A draft care bundle was created and reviewed by members of the respiratory team, the pleural procedures consultant, and the Trust's documentation review committee. In addition to this, electronic written guidance for pleural procedures were produced and reviewed by the respiratory team and Trust's Guidance Committee. The approved "Plan, Do, Study, Act" (PDSA) cycles were used to establish the appearance and content of the final care bundle, guidance, and educational interventions.

PDSA Cycle 1:

The initial results of the pre-intervention audit were used alongside BTS "best practice" recommendations to identify crucial areas needed on a care bundle. An initial draft of the bundle tool was produced using these factors.

PDSA Cycle 2:

The draft care bundle was introduced to the respiratory ward and used to record several drain insertions. Feedback was collected from acute medical registrars and respiratory team members. Reviewers were invited to provide feedback regarding several different areas including appearance, usability, organisation of information, and content. This highlighted areas of improvement from the main users. One user mentioned that the space for documenting rate of drainage from the drain was small and could be lost in the rest of the document. The form was updated to reflect these factors.

PDSA Cycle 3:

The second draft care bundle was then introduced and reviewed over a week by a mixture of medical registrars, junior doctors, and respiratory physicians. Further feedback was obtained about the contents, structure, and usability of the form. Another user mentioned that not every patient undergoing drain insertion required clotting and platelets checked. The form was updated and this process was repeated two further times.

PDSA Cycle 4: 
The care bundle was then reviewed by the Trust's documentation committee and respiratory specialist physicians. The documentation committee wished for a space on the bundle to be created for "nonverbal consent" so this was included to enable Trust wide use. The respiratory consultants also felt that a space for documenting any supervising physician would also be appropriate so this was included. This process was repeated over 15 times over a period of several months until all reviewers were satisfied with the overall content, layout, and appearance of the care bundle.

PDSA Cycle 5:

The finalised care bundle (Appendix 2) was introduced and publicised via brief presentations and posters in the acute care unit, respiratory, and oncology wards. Copies of the forms were kept in an easily accessible location on each of these wards and copies were also filed with the equipment for chest drain insertion. This was trialled over a further period of three months and prospectively audited using the same standardised data collection tool as before (Appendix 1). This data was analysed and the results compared to both the pre-intervention audit and results from the 2014 BTS National Pleural Procedures Audit [9].

PDSA Cycle 6:

It was noted that Gloucestershire Hospitals NHS Trust had no formal guidance or training programme for pleural procedures. Draft guidance for pleural procedures including guidance on training, indications, ultrasonography, location and timing of procedure, consent, asepsis, key points from aspiration and drain insertion techniques, and recognition and management of complications was produced. This aims to provide a more detailed reference for doctors to aid safety of pleural procedures and also provide the foundations for a more formal training programme.

PDSA Cycle 7:

The guidance was reviewed by respiratory consultants and specialist registrars. Feedback was invited on both content, organisation, and appearance. All users were happy with the organisation and presentation of material. Further material regarding the use of suction and management of complications was suggested.

PDSA Cycle 8:

The guidance underwent further review by respiratory physicians and the Trust's Guidance Committee. In total, over eight further subsequent drafts were reviewed before all members were satisfied with the content. A final version of the guidance was then produced (Appendix 3).

PDSA Cycle 9:

The results of the care bundle were presented to both the respiratory team and at the Trust's annual Quality Improvement Initiative in July 2015. The project won first prize due to the improvements it has helped to achieve locally in comparison with national standards. It is now officially in use across Cheltenham General and Gloucester Royal Hospitals. The supportive guidance was also publicised and uploaded to accessible locations on the Trust intranet and treatment guidelines pages. The respiratory team have also secured a chest drain mannequin for formal training of drain insertion and a standardised teaching programme included in Foundation Doctor Training is planned, to help sustain the positive changes which the chest drain bundle has initiated. Re-audit is planned for a further three month period in 2016.

\section{Results}

The data collected on the audit measures described previously was analysed for statistical significance using Fisher's exact test. The data set included 24 pre-intervention and 23 post-intervention (see Figure 1).

Prior to the intervention, documentation was found to be poor; especially in areas related to consent, use of ultrasonography, premedication, post-procedure advice, details regarding length and size of drain and investigations requested. Overall the results showed improvement in most areas of documentation. The results were also compared with the national figures from the most recent national pleural procedures audit [9].

After the introduction of the bundle, all cases had the date documented and $87 \%$ had the time recorded. This showed a significant improvement from pre-intervention levels $(p=0.049)$. Again, $100 \%$ of indications were documented. Overall, there was no significant improvement in the proportion of patients given premedication. However, there was a significant improvement in documenting the rationale for not giving premedication when the bundle was used $(p=0.033)$.

The number of cases where the consent obtained was recorded improved with the bundle but this did not achieve statistical significance. Similarly, there was a nonsignificant improvement in the documentation of ultrasonographic guidance. Both of these measures are better than the national average when the proforma is used. Where the bundle was used, there was a significant improvement in chest drain site documentation $(p=0.0214)$. Documentation of local anaesthetic use was also significantly improved, being recorded in $95.7 \%(p=0.0226)$. Documentation of drain size or method of securing improved but this did not achieve statistical significance. However, there was a significant improvement in the documentation of drain length $(p=0.0145)$.

The bundle did not lead to an improvement in post procedure advice documentation. The use of chest drain observation sheets was unchanged significantly, nor was the documentation of post insertion CXR interpretations, nor was the record of samples sent for investigation.

However only 9 of the 23 (39\%) of drain insertions reviewed used the new bundle. The majority of those that did not use the bundle were performed in clinical areas not targeted by the improvement work; particularly in the emergency department, theatres, and intensive care. When the bundle was used it demonstrated 
improvement in documentation compared to the classical "freehand" documentation.

The percentage of patients who were cared for on a respiratory ward and had a chest drain observation chart post-intervention where much better than the national average (see graphs appendix).

See supplementary file: ds6008.pdf - "figures"

\section{Lessons and limitations}

Introduction of the documentation tool as part of the chest drain bundle has helped improve documentation of chest drain insertion in medicine. In addition to this, feedback from staff has been positive in enabling ease of documentation, reflecting on the procedure and prompting the check of pertinent factors prior to the procedure. This demonstrates the use of a valid insertion proforma to enable uniformity of documentation and protect patients.

However, one key limitation of this work was the patchy use of the bundle. There are likely several reasons for this intermittent use. Several of the physicians targeted by educational interventions rotated midway through the data collection period and their replacements were not updated regarding the bundle. Similarly, several of the clinical areas not targeted demonstrated to insert chest drains more frequently than expected. In the absence of the bundle and targeted education it is not unexpected that results from these areas have not improved in line with the other clinical areas where the bundle was used. This was likely exacerbated as the bundle and guidance was not included on the widely used treatment guidelines on the Trust intranet where many other similarly important documents can be found. There is also the possibility that some drains were missed from this data collection period if the practitioner failed to notify the data collection team.

In the future, the documentation tool will be distributed on a hospital wide basis by the Trust; both in physical form in all places where drains are inserted as well as in an easy to use location on the intranet as part of the care bundle. The comprehensive and easy to read pleural procedures guidance will also be made available to all clinical staff and features links to the insertion bundle. The use of the intranet and accessing guidance is included in departmental induction and so this will help ensure universal exposure to the bundle, education and awareness. The above, coupled with a training event integrated into foundation doctors induction will help ensure that the chest drain bundle leads to sustainable changes.

Education of trainees was not the primary focus of this work. However, given that the improvement procedure does include an educational component, it would be interesting for future work to examine the effect education has on learner satisfaction as well as behaviour.

Another limitation is that it has not been possible to directly observe clinicians inserting chest drains. In this case, ideal outcome measures would include numbers of patients receiving best-practice care (cared on respiratory ward, written informed consent, use of ultrasonography, reduction in inappropriate drains) as well as patient and user satisfaction and comparison of complication rates. Unfortunately, there are several barriers to this. These include the need for longer follow up to assess complication rates, investigator costs, and the requirement for validated questionnaires to fully examine patient and user satisfaction. These all feed in to the most significant barrier to formally observing all inserted chest drains for robust data gathering: that of time.

Due to these significant barriers, quality of documentation has been used as a surrogate marker for changes in behaviour. This was deemed a valid approach for several reasons. An old adage in medicolegal advice states that "if it isn't documented in the notes, it hasn't happened." Legally, failure to document relevant data can be considered a significant breach of and deviation from the standard of care. Record keeping is therefore paramount and thus was deemed a suitable source for data gathering. Critically, existing work suggests that quality of documentation is a valid marker for overall quality of care [10-12]. As such, this was used for this work as a real world measure of procedure quality.

\section{Conclusion}

When used, the chest drain insertion bundle helps improve the documentation of this important procedure. This is important to ensure uniformity in clinical "best" practice, aid communication, and protect patients. This is being implemented across the Trust and with the aid of greater publication and increasingly accessible guidance should help further improve practice.

\section{References}

1 Luketich JD, Kiss M, Hershey J, Urso GK, Wilson J, Bookbinder $M$, Ginsberg R. Chest tube insertion: a prospective evaluation of pain management. Clinical Journal of pain. 1998; 14(2): 152-154.

2 Harris A, O'Driscoll BR, Turkington PM. Survey of major complications of intercostal chest drain insertion in the UK. Journal of Postgraduate medicine. 2010; 86: 68-72.

3 Havelock T, Teoh R, Laws D. On behalf of the British Thoracic Society Pleural disease Group. British Thoracic Society Guideline for Pleural Procedures. Thorax 2010, 65(2): 61-76.

4 Hooper C, Bucknall C, Welham SA, Reid K, Routh C, Maskell NA. Pilot Pleural Procedures Audit. British Thoracic Society, 2009. Available at: https://www.brit-thoracic.org.uk/Portals/0/Audit\%20Tools/pleural au dit_2009_results\%20.pdf (accessed on 27/4/2014)

5 Laws D, Neville E, Duffy J. On behalf of the British Thoracic Society Pleural Disease Group. BTS guidelines for the insertion of a chest drain. Thorax, 2003; 58(2): 53-59.

6 Resar R, Griffin FA, Haraden C, Nolan TW. Using Care Bundles to Improve Health Care Quality. IHI Innovation Series white paper. Cambridge. Massachusetts: Institute for Healthcare Improvement, 
2012. (Available at: www.IHI.org.)

7 Calvert J, Shen Lim W, Rodrigo C, Turner A, Welham S. The British Thoracic Society Pilot Care Bundle Project: A Care BundlesBased Approach to Improving Standards of Care in Chronic Obstructive Pulmonary Disease and Community Acquired Pneumonia. British Thoracic Society Reports, 2014; 6(4).

8 Robb E, Jarman B, Suntharalingam G, Higgens C, Tennant R, Elcock K. Using care bundles to reduce in-hospital mortality: quantitative survey. BMJ, 2010; 340: 1234.

9 Hooper C. British Thoracic Society National Pleural Procedures Audit Report, National Audit Period: 1 June - 31 July 2014. British Thoracic Society Audit Report June 2015 (unpublished).

10 Dunlay S, Alexander K, Melloni C, Kraschnewski J, Liang L, Gibler B, Roe M, Ohman E, Peterson E. Medical records and quality of care in acute coronary syndromes: results from CRUSADE. Archives of Internal Medicine, 2008; 168(15): 1692-1698.

11 Glavan B, Engelberg R, Downey L, Randall Curtis J. Using the medical record to evaluate the quality of end-of-life care in the intensive care unit. Critical care medicine, 2008; 36(4): 1138-1146.

12 Darling S, Sammer M, Chapman T, Parisi M. Physician documentation of fluoroscopy time in voiding cystourethrography reports correlates with lower fluoroscopy times: a surrogate marker of patient radiation exposure. Journal of Roentgenology, 2011;

196(6): 777-780.

\section{Declaration of interests}

None declared.

\section{Acknowledgements}

Thanks to Dr Henry Steer, Dr Zafeiris Sardelis, Dr Claire McBrien, Dr Christopher Williams, and Dr Lucy Duggal.

\section{Ethical approval}

Not required according to local standards. 